\title{
THE CONVERGENCE OF MEASURES ON PARAMETRIC SURFACES
}

\author{
BY \\ J. H. MICHAEL
}

1. Introduction. In [2], H. Federer has proved several theorems, one of which is the following. Let $X$ be a compact differentiable $k$-manifold of class $\infty$ and $f$ be a continuous map of $X$ into $R^{n}$. To each smooth map $f_{i}$ of $X$ into $R^{n}$ corresponds a measure $\mu_{i}$ over the middle space $M_{f}$ of $f$, whose values are $k$-dimensional currents in $R^{n}$. This measure associates with any continuous, real-valued function $\chi$ on $M_{f}$ the current $f_{i \#}\left\{X \wedge\left(\chi \circ m_{f}\right)\right\}$ given by the formula

$$
f_{i \#}\left\{X \wedge\left(\chi \circ m_{f}\right)\right\}(\phi)=\int_{X}\left(\chi \circ m_{f}\right) \wedge f_{i}^{\#}(\phi),
$$

whenever $\phi$ is a differential $k$-form of class $\infty$ on $R^{n} . m_{f}$ denotes the monotone component in the monotone light factorization of $f$.

If $f$ has finite Lebesgue area and either $k=2$ or the range of $f$ has $k+1$ dimensional Hausdorff measure zero, then there exists a unique current valued measure $\mu$ over $M_{f}$ such that for every sequence of smooth maps $f_{i}$, which converge uniformly to $f$ and whose areas are bounded, the measures $\mu_{i}$ converge weakly to $\mu$.

Federer also treats the case where $X$ is not compact but $f$ is proper; i.e., $f^{-1}(Y)$ is compact for every compact $Y \subset R^{n}$.

It is the purpose of this paper to generalise this theorem (except for the special case $k=2$ ) to cover the case of a manifold with boundary. (Actually, an oriented pseudo-manifold is used.) The following result is obtained.

Let $M$ be a $k$-dimensional pseudo-manifold $(k \geqq 1)$ and $C$ an integral $k$-chain on $M$ such that the support of $\partial(c)$ is the boundary of $M$. Let $f$ be a continuous mapping of $|M|$ into $R^{n}$ with middle space $\mathscr{M}$ and monotone light factorization $f=l_{f} \circ m_{f}$. As with Federer's smooth maps, there corresponds to each quasi-linear mapping $f_{i}$ of $|M|$ into $R^{n}$, a measure $\mu_{i}$ over $M$ whose values are $k$-dimensional currents in $R^{n}$. For each continuous function $\chi$ on $M$,

$$
\left\{\mu_{i}(\chi)\right\}(\phi)=\sum_{\sigma} \int_{A_{\sigma}} c\left(T_{\sigma}\right) \cdot \chi\left[m\left\{T_{\sigma}(\chi)\right\}\right] \wedge\left[\left(f_{i} \circ T_{\sigma}\right)^{\#}(\phi)\right]
$$

where $\phi$ is a differential $k$-form of class $\infty$ on $R^{n}$, the summation is taken over all $k$-simplexes $\sigma$ of $M$, each $T_{\sigma}$ is a linear nonsingular mapping of a subset $A_{\sigma}$ of

Presented to the Society, January 26, 1962; received by the editors June 20, 1961 and, in revised form, April 13, 1962. 
$R^{k}$ onto $\sigma$ and $c\left(T_{\sigma}\right)$ is the coefficient of the chain $c$ on the orientation of $\sigma$ corresponding to $T_{\sigma}$.

If $f$ has finite Lebesgue area and the range of $f$ has $k+1$ dimensional Hausdorff measure zero, then there exists a unique current valued measure $\mu$ over $\mathscr{M}$ such that for every sequence of quasi-linear mappings $f_{i}$ which converge uniformly to $f$ and whose elementary areas converge to the Lebesgue area of $f$, the measures $\mu_{i}$ converge weakly to $\mu$.

No attempt is made to generalise the other theorems of Federer's paper.

2. Preliminaries. Throughout the paper we are concerned with (finite) geometric complexes and their integral chains. The definition adopted for a geometric complex will be that of Pontrjagin [8], in which the simplexes are closed sets.

We follow Lefschetz [5] in defining subdivisions; i.e., we define $K_{1}$ to be a subdivision of $K$ if $\left|K_{1}\right|=|K|$ and for every simplex $\zeta$ of $K_{1}$, there exists a simplex $\sigma$ of $K$ containing $\zeta$.

As in Whitney [9], a pseudo-manifold of dimension $k$ (where $k \geqq 1$ ) is a geometric complex $K$ with the following two properties:

(i) every simplex of $K$ is a face of at least one $k$-simplex;

(ii) every $(k-1)$-simplex is a face of at most two $k$-simplexes.

No connectivity properties are assumed. The boundary $\mathscr{B}(K)$ of $K$ is the subcomplex of $K$ formed by the closure of the set of all those $(k-1)$-simplexes, each of which is a face of only one $k$-simplex.

2.1. QUASI-LINEAR MAPPINGS. For the purposes of this paper a quasi-linear mapping of a geometric complex $L$ into a euclidean space $R^{n}$ is a continuous mapping $g$ of $|L|$ into $R^{n}$ for which there exists a subdivision $L_{1}$ of $L$ with $g$ linear on each simplex of $L_{1}$. Clearly

2.1.1. If $L_{*}$ is a geometric complex with $\left|L_{*}\right| \subseteq|L|$, then the restriction of $g$ to $\left|L_{*}\right|$ is quasi-linear with respect to $L_{*}$.

2.2. INDUCED MEASURES. Let $K$ be a geometric complex and $c$ a nonzero integral $k$-chain on $K(k \geqq 1)$. Denote by $M$ and $N$ the sub-complexes of $K$ that support $c$ and $\partial(c)$ respectively. Let $g$ be a quasi-linear mapping of $M$ into $R^{n}$. We associate with $g$, in the following way, a measure $v(g)$ on the space $C$ of realvalued continuous functions on $|M|$ whose values are $k$-dimensional currents in $R^{n}$. For each $\psi \in C$ and each differential $k$-form $\phi$ of $R^{n}$ of class ( $)$,

$$
[\{v(g)\}(\psi)](\phi)=\sum_{\sigma} \int_{A_{\sigma}} c\left(T_{\sigma}\right) \cdot \psi\left[T_{\sigma}(x)\right] \wedge\left[\left(g \circ T_{\sigma}\right)^{\#}(\phi)\right] d x,
$$

where the summation is taken over all $k$-simplexes $\sigma$ of $M$, each $T_{\sigma}$ is a linear nonsingular mapping of a subset $A_{\sigma}$ of $R^{k}$ onto $\sigma$ and $c\left(T_{\sigma}\right)$ is the coefficient of the chain $c$ on the orientation of $\sigma$ given by the mapping $T_{\sigma}$. \{The notation in the above formula is that of [3].\} 
If $B$ is a Borel subset of $|M|$, then the elementary area of $g$ on $B$ is

$$
a(g, B)=\sum_{\sigma^{k} \in M_{1}}\left[\sum_{P \in P_{k}^{n}}\{k \text {-dimensional measure of }(P \circ g)(B \cap \sigma)\}^{2}\right]^{1 / 2}
$$

where $M_{1}$ is a subdivision of $M$ such that $g$ is linear on each simplex of $M_{1}$ and $P_{k}^{n}$ denotes the collection of all those projections from $R^{n}$ to $R^{k}$ formed by deleting $n-k$ of the coordinates.

2.3. Lebesgue area. Let $K, c, M$ and $N$ be as in 2.2. Let $f$ be a continuous mapping of $|M|$ into $R^{n}$. For the purposes of this paper, the Lebesgue area of $f$ will be

$$
L(f)=\inf \lim _{r \rightarrow \infty} \inf a\left(f^{(r)},|M|\right),
$$

where the infimum is taken over all sequences $\left\{f^{(r)}\right\}$ of quasi-linear mappings of $M$ into $R^{n}$ such that $f^{(r)} \rightarrow f$ uniformly as $r \rightarrow \infty$.

3. Retraction of the boundary. In this section we prove a theorem to the effect that the boundary of a surface with finite Lebesgue area makes only a small contribution to the area.

We will be dealing with geometric complexes and the following notation is adopted. $\Pi(K)$ denotes the barycentric subdivision of a geometric complex $K$. We denote the closure of a collection $L$ of simplexes of $K$ by $\mathrm{Cl}(L)$. Of the several definitions in use for the star $\operatorname{St}(\sigma)$ of a simplex $\sigma$ of $K$ we select the one that defines $\operatorname{St}(\sigma)$ to be the collection of all these simplexes with $\sigma$ for a face ( $\sigma$ being regarded as a face of itself). $K^{r}$ will denote the $r$-dimensional skeleton of $K$.

3.1. Pseudo-manifolds. We obsesve that a pseudo-manifold $K$ has the following properties:

3.1.1. If $\operatorname{dim} k \geqq 1$, then either $\mathscr{B}(K)=\varnothing$ or $\operatorname{dim} \mathscr{B}(K)=\operatorname{dim} K-1$.

3.1.2. $\Pi(K)$ is a pseudo-manifold and $\mathscr{B}\{\Pi(K)\}=\Pi\{\mathscr{B}(K)\}$.

3.2. Definition. We define an $F$-complex to be a nonempty pseudo-manifold $K$ of dimension $k \geqq 1$ and such that: for every pair $\sigma_{1}^{k}, \sigma_{2}^{k}$ of distinct $k$-simplexes of $K$, there exists a sequence

$$
\sigma_{1}^{k}=\xi_{0}^{k}, \xi_{1}^{k}, \cdots, \xi_{r}^{k}=\sigma_{2}^{k}
$$

of distinct $k$-simplexes of $K$ such that for each $i=1, \cdots, r, \xi_{i-1}$ and $\xi_{i}$ have a common $(k-1)$-face.

Evidently

3.2.1. If $K$ is an $F$-complex, then $\Pi(K)$ is also an $F$-complex.

3.3. Definition. Let $K$ be a pseudo-manifold with dimension $k \geqq 1$. There exists a unique set $K_{1}, K_{2}, \cdots, K_{p}$ of subcomplexes of $K$ with the following properties:

(i) each $K_{i}$ is an $F$-complex, 
(ii) no two of $K_{1}, K_{2}, \cdots, K_{p}$ have a $k$-simplex or a $(k-1)$-simplex in common;

(iii) $K=K_{1} \cup K_{2} \cdots \cup K_{p}$.

$K_{1}, K_{2}, \cdots, K_{p}$ will be called the $F$-components of $K$.

Evidently

3.3.1. $\mathscr{B}(K)=\mathscr{B}\left(K_{1}\right) \cup \mathscr{B}\left(K_{2}\right) \cup \cdots \cup \mathscr{B}\left(K_{p}\right)$.

3.4. Lemma. If $K$ is an F-complex of dimension $k \geqq 1$ and $\sigma^{k-1}$ is $a(k-1)$ simplex of $\mathscr{B}(K)$, then there exists a continuous mapping $\phi$ of $|K|$ into $\left|K^{k-1}\right|$ such that

$$
\phi(x)=x
$$

for all $x \in\left|\mathscr{B}(K) \sim \sigma^{k-1}\right| \cup\left|K^{k-2}\right|$.

Proof. Since $K$ is an $F$-complex we can arrange its $k$-simplexes into a sequence

$$
\sigma_{1}^{k}, \sigma_{2}^{k}, \cdots, \sigma_{p}^{k}
$$

in such a way that $\sigma^{k-1}$ is a face of $\sigma_{1}^{k}$ and for $i=2,3, \cdots, p, \sigma_{i}^{k}$ has a $(k-1)$-face $\sigma_{i}^{k-1}$ in common with one of $\sigma_{1}^{k}, \sigma_{2}^{k}, \cdots, \sigma_{i-1}^{k}$. Put $\sigma_{1}^{k-1}=\sigma^{k-1}$. For each $i=1,2, \cdots, p$, let $K_{i}$ be the subcomplex obtained by removing $\sigma_{1}^{k}, \sigma_{2}^{k}, \cdots, \sigma_{i}^{k}$, $\sigma_{1}^{k-1}, \cdots, \sigma_{i}^{k-1}$ from $K$. Put $K_{0}=K$.

For each $i=1, \cdots, p$ there exists a continuous mapping $\psi_{i}$ of $\sigma_{i}^{k}$ into $\left|\mathscr{B}\left(\sigma_{i}^{k}\right) \sim \sigma_{i}^{k-1}\right|$ such that $\psi_{i}(x)=x$ for all $x \in\left|\mathscr{B}\left(\sigma_{i}^{k}\right) \sim \sigma_{i}^{k-1}\right|$. Define

$$
\begin{aligned}
\phi_{i}(x) & =\psi_{i}(x) \text { for } x \in \sigma_{i}^{k} \\
& =x \text { for } x \in\left|K_{i-1}\right| \sim \sigma_{i}^{k} .
\end{aligned}
$$

Then $\phi_{i}$ is a continuous mapping of $\left|K_{i-1}\right|$ into $\left|K_{i}\right|$ such that $\phi_{i}(x)=x$ for all $x \in\left|K_{i}\right|$. Define

$$
\phi=\phi_{p} \phi_{p-1} \cdots \phi_{1} \text {. }
$$

Then $\phi$ is a continuous mapping of $\left|K_{0}\right|=|K|$ into $\left|K_{p}\right| \subseteq\left|K^{k-1}\right|$ such that

$$
\phi(x)=x
$$

for all $x \in\left|K_{p}\right| \supseteq\left|\mathscr{B}(K) \sim \sigma^{k-1}\right| \cup\left|K^{k-2}\right|$.

3.5. Lemma. Let $K$ be a pseudo-manifold of dimension $k \geqq 1$ and let $L$ be a sub-pseudo-manifold of $K$ such that each F-component of $L$ has a $(k-1)$ simplex in common with $\mathscr{B}(K)$.

There exists a continuous mapping $\psi$ of $|K|$ into $|K|$ and a subdivision $\Pi^{r}(K)$ of $K$ such that

(i) $\psi(|L|) \subseteq\left|L^{k-1}\right|$;

(ii) $\psi(x)=x$ for all $x \in|\mathrm{Cl}(K \sim L)|$ and

(iii) for each $k$-simplex $\xi^{k}$ of $\Pi^{r}(L)$, there exists a $(k-1)$-simplex $\sigma^{k-1}$ of $L^{k-1}$ with $\psi\left(\xi^{k}\right) \subseteq \sigma^{k-1}$ and with $\psi$ linear on $\xi^{k}$. 
Proof. Let $L_{1}, L_{2}, \cdots, L_{p}$ be the $F$-components of $L$. Let $\sigma_{i}^{k-1}$ be a $(k-1)$ simplex that $L_{i}$ has in common with $\mathscr{B}(K)$. Then $\sigma_{i}^{k-1} \in \mathscr{B}\left(L_{i}\right)$, hence by 4.4, there is a continuous mapping $\phi_{i}$ of $\left|L_{i}\right|$ into $\left|L_{i}^{k-1}\right|$ such that

$$
\phi_{i}(x)=x
$$

for all $x \in\left|\mathscr{B}\left(L_{i}\right) \sim \sigma_{i}^{k-1}\right|$. Define

$$
\begin{aligned}
\phi(x) & =x & & \text { if } x \in|\mathrm{Cl}(K \sim L)| \\
& =\phi_{i}(x) & & \text { if } x \in L_{i} .
\end{aligned}
$$

Then $\phi$ is a continuous mapping of $|K|$ into $|K|$ and

$$
\phi(|L|) \subseteq\left|L^{k-1}\right| \text {. }
$$

Choose a positive integer $r$ such that for every vertex $x$ of $\Pi^{r}(L)$, there exists a vertex $y$ of $L^{k-1}$ with

$$
\phi\left\{\mid \operatorname{St}(x) \text { in } \Pi^{r}(L) \mid\right\} \subseteq S(y)
$$

where $S(y)$ denotes the union of the interiors of the simplexes of $\operatorname{St}(y)$ in $L^{k-1}$. Define a function $\psi$ from $\left\{\Pi^{r}(L)\right\}^{0}$ to $|L|$ such that

$$
\psi(x)=x
$$

if $x \in\left\{\Pi^{r}(L)\right\}^{0} \cap|\mathrm{Cl}(K \sim L)|$ and such that when $x \in\left\{\Pi^{r}(L)\right\}^{0} \sim|\mathrm{Cl}(K \sim L)| \psi(x)$ is a vertex of $L^{k-1}$ with

$$
\phi\left\{\mid \operatorname{St}(x) \text { in } \Pi^{r}(L) \mid\right\} \subseteq S\{\psi(x)\} .
$$

We will now prove that if $v_{0}, v_{1}, \cdots, v_{k}$ are the vertices of a $k$-simplex of $\Pi^{r}(L)$, then there exists a simplex $\sigma$ of $L^{k-1}$ such that

$$
\psi\left(v_{0}\right), \cdots, \psi\left(v_{k}\right) \in \sigma .
$$

Since $r \geqq 1$, there is at least one $v_{i}$ not in $|\mathrm{Cl}(K \sim L)|$. If no $v_{i}$ is in $|\mathrm{Cl}(K \sim L)|$, then (6) follows immediately from (5). If $v_{0}, \cdots, v_{s}$ are in $|\mathrm{Cl}(K \sim L)|$ but $v_{s+1}, \cdots, v_{k}$ are not, then by (4), there exists a simplex $\sigma_{1}$ of $L^{k-1}$ containing $\psi\left(v_{0}\right), \cdots, \psi\left(v_{s}\right)$. But

so that by (5)

$$
v_{0} \in \mid \operatorname{St}\left(v_{j}\right) \text { in } \Pi^{r}(L) \mid
$$

$$
\phi\left(v_{0}\right) \in S\left\{\psi\left(v_{j}\right)\right\}
$$

for $j=s+1, \cdots, k$; hence since $\phi\left(v_{0}\right)=v_{0}$ it follows from (4) that

Then

$$
\psi\left(v_{0}\right) \in S\left\{\psi\left(v_{j}\right)\right\}, \quad j=s+1, \cdots, k .
$$

$$
\sigma_{1} \in \operatorname{St}\left\{\psi\left(v_{j}\right)\right\} \text { in } L^{k-1}
$$

for $j=s+1, \cdots, k$; i.e., each $\psi\left(v_{j}\right)$ is a vertex of $\sigma$. Thus (6) is true. 
We can now extend $\psi$ to a continuous mapping of $|K|$ into $|K|$ by putting

$$
\psi(x)=x
$$

for all $x \in|\mathrm{Cl}(K \sim L)|$ and extending $\psi$ linearly over each $k$-simplex of $\Pi^{r}(L)$. It follows immediately from (6) and (7), that $\psi$ has the required properties.

3.6. THEOREM. Let $K$ be a pseudo-manifold of dimension $k \geqq 1$ and $c$ be a nonzero integral $k$-chain on $K$ such that $\partial(c)$ is supported by $\mathscr{B}(K)$. Let $f$ be a continuous mapping of $|K|$ into $R^{n}(n \geqq k)$ with finite Lebesgue area and $\left\{f^{(r)}\right\}$ be a sequence of quasi-linear mappings of $K$ into $R^{n}$ such that

and

$$
\left\|f^{(r)}-f\right\| \rightarrow 0
$$

$$
a\left(f^{(r)},|K|\right) \rightarrow L(f)
$$

as $r \rightarrow \infty$. Let $\varepsilon>0$ and $B$ be a dense subset of $R^{1}$.

There exists a finite number $J_{1}, \cdots, J_{p}$ of closed intervals of $R^{n}$ such that:

(i) $J_{1}, \cdots, J_{p}$ have mutually disjoint interiors and the coordinates of their vertices are all in $B$;

$$
f(|\mathscr{B}(K)|) \subseteq \operatorname{Int}\left\{\bigcup_{j=1}^{p} J_{j}\right\} ;
$$

(iii) for every $\delta>0$, there exists a subdivision $\Pi^{l}(K)$ of $K$ and a sub-pseudomanifold $L$ of $\Pi^{l}(K)$ such that every point of $f(|\mathscr{B}(L)|)$ has a distance less than $\delta$ from $\bigcup_{j=1}^{p} \operatorname{Fr}\left(J_{j}\right)$ and

$$
\underset{r \rightarrow \infty}{\limsup } a\left(f^{(r)},|K| \sim|L|\right)<\varepsilon .
$$

Proof. We can construct an infinite sequence $\mathscr{J}_{1}, \mathscr{J}_{2}, \cdots$ with the following property:

(A) each $\mathscr{J}_{t}$ is a finite collection of closed intervals of $R^{n}$ with mutually disjoint interiors, with the coordinates of all their vertices in $B$, with diameters less than $1 / t$, with

$$
f\{|\mathscr{B}(K)|\} \subseteq \operatorname{Int}\left\{\bigcup_{J \in \mathscr{I} t} J\right\}
$$

and all intersecting $f\{|\mathscr{B}(K)|\}$.

For each $J \in \mathscr{J}_{t}$ let $V_{J}$ be the set consisting of all those points $x$ of $f^{-1}(\operatorname{Int} J)$ with the property:

(B) there exists a subdivision $\Pi^{v}(K)$ of $K$ and a subcomplex $M$ of $\Pi^{v}(K)$ such that $M$ is an $F$-complex with a $(k-1)$-simplex in common with $\mathscr{B}\left\{\Pi^{v}(K)\right\}=\Pi^{v}\{\mathscr{B}(K)\}$ and $x \in|M| \subseteq f^{-1}(\operatorname{Int} J)$.

For each $r$, let $K_{r}$ be a subdivision of $K$ such that $f^{(r)}$ is linear on each simplex of $K_{r}$. A point $x$ belongs to $V_{J}$ if and only if 
(C) for each $r$, there exists a subdivision $\Pi^{v_{r}}\left(K_{r}\right)$ and a subcomplex $M$ of $\Pi^{v_{r}}\left(K_{r}\right)$ such that $M$ is an $F$-complex with a $(k-1)$-simplex in common with $\mathscr{B}\left\{\Pi^{v_{r}}\left(K_{r}\right)\right\}=\Pi^{v_{r}}\left\{\mathscr{B}\left(K_{r}\right)\right\}$ and $x \in|M| \subseteq f^{-1}(\operatorname{Int} J)$.

Put

$$
V_{t}=\bigcup_{J \in \mathscr{J}_{t}} V_{\mathrm{J}}
$$

We will prove that

$$
\lim _{t \rightarrow \infty}\left[\limsup _{r \rightarrow \infty} a\left\{f^{(r)}, V_{t}\right\}\right]=0
$$

To prove (2), we suppose it is not true. Then there exists an $\varepsilon^{\prime}>0$ and two subsequences $\left\{r_{s}\right\},\left\{t_{s}\right\}$ of the positive integers such that

$$
a\left\{f^{\left(r_{s}\right)}, V_{t_{s}}\right\}>\varepsilon^{\prime}
$$

for all $s=1,2, \cdots$. For all positive integers $q, s$ and all $J \in \mathscr{J}_{t_{c}}$, let $L_{q s}(J)$ denote the sub-pseudo-manifold of $\Pi^{q}\left(K_{r_{s}}\right)$ that is the closure of the set of all those $k$-simplexes that are contained in $V_{J}$. Let $M_{q s}(J)$ denote the subcomplex of $L_{q s}(J)$ consisting of the union of all those $F$-components of $L_{q s}(J)$ that have a $(k-1)$-simplex in common with $\mathscr{B}\left\{\left(\Pi^{q}\left(K_{r_{s}}\right)\right\}=\Pi^{q}\left\{\mathscr{B}\left(K_{r_{s}}\right)\right\}\right.$. Then

$$
\lim _{q \rightarrow \infty} \bigcup_{J \in \mathscr{J} t_{s}}\left|M_{q s}(J)\right|=V_{t_{-}},
$$

hence by (3) we can choose, for each $s$, a positive integer $q_{s}$ such that

$$
a\left\{f^{\left(r_{s}\right)}, \bigcup_{J \in \mathscr{I}_{t s}}\left|M_{q_{s} s}(J)\right|\right\}>\frac{1}{2} \varepsilon^{\prime} .
$$

By 3.5, there exists for each $J \in \mathscr{J}_{t_{s}}$ a continuous mapping $\psi_{J}$ of $|K|$ into $|K|$ and a subdivision $\Pi^{r_{s}}\left(K_{r_{s}}\right)$ of $\Pi^{q_{s}}\left(K_{r_{s}}\right)$ such that:

(i) $\psi_{J}\left\{\left|M_{q_{s} s}(J)\right|\right\} \subseteq\left|\left\{M_{q_{s} s}(J)\right\}^{k-1}\right|$;

(ii) $\psi_{J}(x)=x$ for all $x \in\left|\mathrm{Cl}\left\{\Pi^{q_{s}}\left(K_{r_{s}}\right) \sim M_{q_{s} s}(J)\right\}\right|$;

(iii) for each $k$ simplex $\xi^{k}$ of $\Pi^{v_{s}-q_{s}}\left\{M_{q_{s} s}(J)\right\}$ there exists a $(k-1)$-simplex $\sigma^{k-1}$ of $\left\{M_{q_{s} s}(J)\right\}^{k-1}$ such that $\psi_{J}\left(\xi^{k}\right) \subseteq \sigma^{k-1}$ and $\psi_{J}$ is linear on $\xi^{k}$.

By forming a composition of the mappings $\psi_{J}\left(J \in \mathscr{J}_{t_{s}}\right)$ and $f^{\left(r_{s}\right)}$, we arrive at a quasi-linear mapping $g^{\left(r_{s}\right)}$ of $K$ into $R^{n}$ with the following properties:

$$
\begin{aligned}
& \left\|g^{\left(r_{s}\right)}-f^{\left(r_{s}\right)}\right\|<\frac{1}{t_{s}} ; \\
& a\left(g^{\left(r_{s}\right)},|K|\right)=a\left(f^{\left(r_{s}\right)},|K| \sim \bigcup_{J \in g_{t_{s}}}\left|M_{q_{s} s}(J)\right|\right) .
\end{aligned}
$$

By $(\alpha)$ and hypothesis

$$
\left\|g^{\left(r_{s}\right)}-f\right\| \rightarrow 0
$$


as $s \rightarrow \infty$ and by $(\beta)$ and (4)

$$
\limsup _{s \rightarrow \infty} a\left(g^{(r s)},|K|\right) \leqq L(f)-\varepsilon^{\prime}
$$

contrary to hypothesis. Thus (2) is true.

By (2) we can choose a positive integer $t^{\prime}$ such that

$$
\underset{r \rightarrow \infty}{\limsup } a\left\{f^{(r)}, V_{t^{\prime}}\right\}<\varepsilon
$$

and we let $J_{1}, \cdots, J_{p}$ be the members of $\mathscr{J}_{t^{\prime}}$.

To prove (iii), take an arbitrary $\delta>0$ and let $C$ be the subset of $|K|$ consisting of all those points $x$ for which $x \in V_{t^{\prime}}$ and $f(x)$ has a distance $\geqq \frac{1}{2} \delta$ from $\bigcup_{j=1}^{p} \operatorname{Fr}\left(J_{j}\right)$. Then $C$ is compact. For each $j=1, \cdots, p$, let $L_{q j}$ be the subcomplex of $\Pi^{q}(K)$ that is the closure of the set of all those $k$-simplexes that are contained in $V_{J_{j}}$. Let $M_{q j}$ denote the subcomplex of $L_{q j}$ consisting of the union of all those $F$-components of $L_{q j}$ that have a $(k-1)$-simplex in common with $\mathscr{B}\left\{\Pi^{q}(K)\right\}$ $=\Pi^{q}\{\mathscr{B}(K)\}$. Let

Then

$$
M_{q}=\bigcup_{j=1}^{p} M_{q j}
$$

$$
\lim _{q \rightarrow \infty}\left[\text { Int of }\left|M_{q}\right| \text { in } V_{t^{\prime}}\right]=V_{t^{\prime}}
$$

hence there exists an $l$ such that $C \subseteq\left|M_{l}\right|$. Put

$$
L=\mathrm{Cl}\left\{\Pi^{l}(K) \sim M_{l}\right\},
$$

and $L$ has the required properties.

4. The main convergence theorem. Throughout $\S 4, K$ will be a geometric complex, $c$ a nonzero integral $k$-chain $(k \geqq 1)$ on $K$ and $M, N$ the subcomplexes of $K$ that support $c$ and $\partial(c)$ respectively. $f$ is a continuous mapping of $|M|$ into $R^{n}$.

$f$ has a unique monotone light factorization (see [2] or [10]),

$$
f=l \circ m m:|M| \rightarrow \mathscr{M} l: \mathscr{M} \rightarrow R^{n},
$$

whose middle space $\mathscr{M}$ consists of the maximal continua of constancy of $f$. $M$ can be metrized by defining

$$
d(\xi, \eta)=\inf \{\operatorname{diam} f(C) ; C \text { is a continuum of }|M| \text { containing } \xi \cup \eta\},
$$

when $\xi, \eta$ both belong to the same component of $|M|$ and

$$
d(\xi, \eta)=1+\operatorname{diam} f(|M|)
$$

otherwise. $\mathscr{M}$ is evidently compact. 
Let $\mathscr{C}$ be the Banach space of all real-valued continuous functions on $\mathscr{M}$ with the norm of uniform convergence.

For each quasi-linear mapping of $g$ of $M$ into $R^{n}$, there was defined in 2.2 a current-valued measure $v(g)$ over $|M|$. This induces a measure $\mu(g)$ over $\mathscr{M}$ as follows:

$$
\{\mu(g)\}(\chi)=\{v(g)\}(\chi \circ m), \quad \chi \in \mathscr{C} .
$$

4.1. THEOREM. If $f$ has finite Lebesgue area, $f(|M|)$ has $k+1$ dimensional Hausdorff measure zero and $\partial(c)=0$, then there exists a unique current-valued measure $\mu(f)$ over $\mathscr{M}$ such that for every sequence $\left\{f^{(r)}\right\}$ of quasi-linear mappings of $M$ into $R^{n}$ which converge uniformly tof and have bounded areas, the measures $\mu\left(f^{(r)}\right)$ converge weakly to $\mu(f)$.

This theorem is just 3.9 of [2] without the special case $k=2$. Although Federer's theorem is stated for differentiable $k$-manifolds of class $\infty$, the proof is valid for $k$-dimensional geometric complexes.

4.2. LemmA. Let $M$ be a pseudo-manifold, $N=\mathscr{B}(M), f$ have finite Lebesgue area and $f(|M|)$ have $k+1$ dimensional Haudsorff measure zero. Let $\mathscr{C}_{0}$ be the subspace of $\mathscr{C}$ consisting of all $\chi \in \mathscr{C}$ for which $\chi \circ m$ vanishes on $|N|$. Then there exists a unique current-valued measure $\mu_{0}(f)$ on $\mathscr{C}_{0}$ such that for every sequence $\left\{f^{(r)}\right\}$ of quasi-linear mappings of $M$ into $R^{n}$ which converge uniformly to $f$ and have bounded areas, the restrictions of the measures $\mu\left(f^{(r)}\right)$ to $\mathscr{C}_{0}$ converge weakly to $\mu_{0}(f)$ on $\mathscr{C}_{0}$.

Proof. Let $M_{*}$ be a $k$-dimensional pseudo-manifold containing $M$ as a subcomplex and such that:

(A) $\mathscr{B}\left(M_{*}\right)=\varnothing$;

(B) if $L=\mathrm{Cl}\left(M_{*} \sim M\right)$, then $M \cap L=N=\mathscr{B}(M)=\mathscr{B}(L)$;

(C) there exists an isomorphism $\theta$ of $|M|$ onto $|L|$ such that $\theta(\sigma)=\sigma$ for all $\sigma \in \mathscr{B}(M)$.

Let $\eta$ denote the corresponding homeomorphism. The chain $c$ extends to an integral $k$-cycle $c_{*}$ on $M_{*}$.

For each continuous mapping $h$ of $|M|$ into $R^{n}$, let $h_{*}$ be the continuous mapping of $\left|M_{*}\right|$ into $R^{n}$ given by

$$
\begin{aligned}
h_{*}(x) & =h(x), & & x \in|M|, \\
& =h\left\{\eta^{-1}(x)\right\}, & & x \in|L| .
\end{aligned}
$$

For a quasi-linear mapping $g:|M| \rightarrow R^{n}$ we evidently have

hence

$$
a\left(g_{*},\left|M_{*}\right|\right)=2 a(g,|M|),
$$

$$
L\left(f_{*},\left|M_{*}\right|\right) \leqq 2 L(f,|M|) \text {. }
$$


Denote the middle space of $f_{*}$ by $\mathscr{M}_{*}$. By 4.1 , there exists a unique measure $\mu_{*}$ over $\mathscr{M}_{*}$ such that for every sequence $\left\{q_{*}^{(r)}\right\}$ of quasi-linear mappings of $\left|M_{*}\right|$ into $R^{n}$, which converge uniformly to $f_{*}$ and have bounded areas, the measures $\mu\left(q_{*}^{(r)}\right)$ converge weakly to $\mu_{*}$. For each $\chi \in \mathscr{C}_{0}$, let $\chi_{*}$ be the continuous function on $M_{*}$ given by

Define

$$
\begin{aligned}
\chi_{*}(\zeta) & =\chi(|M| \cap \zeta) \text { if }|M| \cap \zeta \neq \varnothing, \\
& =0 \text { if }|M| \cap \zeta=\varnothing
\end{aligned}
$$

$$
\left\{\mu_{0}(f)\right\}(\chi)=\mu_{*}\left(\chi_{*}\right), \quad \chi \in \mathscr{C}_{0} .
$$

If $\left\{f^{(r)}\right\}$ is a sequence of quasi-linear mappings of $M$ into $R^{n}$, which converge uniformly to $f$ and have bounded areas, then $f^{(r)}$ converges uniformly to $f_{*}$ and $a\left(f_{*}^{(r)},\left|M_{*}\right|\right)$ is bounded, hence, for each $\chi \in \mathscr{C}_{0}$

so that

$$
\lim _{r \rightarrow \infty}\left\{\mu\left(f_{*}^{(r)}\right)\right\}\left(\chi_{*}\right)=\mu_{*}\left(\chi_{*}\right),
$$

$$
\lim _{r \rightarrow \infty}\left\{\mu\left(f^{(r)}\right)\right\}(\chi)=\left\{\mu_{0}(f)\right\}(\chi) .
$$

4.3. THEOREM. If $M$ is a pseudo-manifold, $N=\mathscr{B}(M), f$ has finite Lebesgue area and $f(|M|)$ has $k+1$ dimensional Hausdorff measure zero, then there exists a unique current-valued measure $\mu(f)$ over $M$ such that, for every sequence $\left\{f^{(r)}\right\}$ of quasi-linear mappings of $M$ into $R^{n}$, which converge uniformly to $f$ and whose areas converge to the Lebesgue area of $f$, the measures $\mu\left(f^{(r)}\right)$ converge weakly to $\mu(f)$.

Proof. Since every uniformly bounded sequence of measures over $\mathscr{M}$ has a convergent subsequence, it will be sufficient to prove that if $\left\{g^{(r)}\right\},\left\{h^{(r)}\right\}$ are two sequences of quasi-linear mappings of $M$ into $R^{n}$, which converge uniformly to $f$, whose areas converge to the Lebesgue area of $f$ and which are such that the corresponding sequences of measures $\left\{\mu\left(g^{(r)}\right)\right\},\left\{\mu\left(h^{(r)}\right)\right\}$ converge weakly to measures $\mu$ and $\mu^{\prime}$, then $\mu=\mu^{\prime}$.

Suppose this statement is not true, i.e., there exists a $\chi \in \mathscr{C}$ such that $\mu(\chi) \neq \mu^{\prime}(\chi)$. Then

$$
\left|\lim _{r \rightarrow \infty}\left\{v\left(g^{(r)}\right)\right\}(\chi \circ m)-\lim _{r \rightarrow \infty}\left\{v\left(h^{(r)}\right)\right\}(\chi \circ m)\right|=5 \varepsilon>0 .
$$

Put

$$
K=1+\sup _{\zeta \in \mathscr{M}} \chi(\zeta)
$$

We can assume that there exists a countable dense subset $Y$ of $R^{1}$ such that if we put for $i,=1, \cdots, n$ and $t \in Y$

$$
A_{i}=\left\{x ; x \in R^{n} \text { and } x_{i}<t\right\},
$$


then each of the limits

$$
\begin{aligned}
& \lim _{r \rightarrow \infty} a\left\{g^{(r)}, f^{-1}\left(A_{i}\right)\right\}=\rho_{i}(t) \\
& \lim a\left\{h^{(r)}, f^{-1}\left(A_{i}\right)\right\}=\rho_{i}^{*}(t)
\end{aligned}
$$

exists; because, if not, we could choose subsequences for which these limits existed. Since $\rho_{i}$ and $\rho_{i}^{*}$ are monotone increasing there exists a subset $Z$ of $R^{1}$ such that $R^{1} \sim Z$ has zero measure and $\rho_{i}^{\prime}(t), \rho_{i}^{* \prime}(t)$ exist for all $t \in Z$ amd all $i$. Then, for every interval $J$ of $R^{n}$ with the coordinates of its vertices all in $Z$, we have

$$
\lim _{\lambda \rightarrow 0+} \limsup _{r \rightarrow \infty}\left[a\left\{g^{(r)}, f^{-1}\left(B_{\lambda}\right)\right\}+a\left\{h^{(r)}, f^{-1}\left(B_{\lambda}\right)\right\}\right]=0,
$$

where

$$
B_{\lambda}=\left\{x ; x \in R^{n} \text { and } d[x, \operatorname{Fr}(J)]<\lambda\right\} .
$$

By 3.6, there exists a finite number $J_{1}, \cdots, J_{p}$ of closed intervals of $R^{n}$ such that (A) $J_{1}, \cdots, J_{p}$ have mutually disjoint interiors and the coordinates of their vertices are all in $Z$;

$$
f(|N|) \subseteq \operatorname{Int}\left\{\sum_{j=1}^{p} J_{j}\right\} ; \text { and }
$$

(C) for every $\delta>0$, there exists a subdivision $\Pi^{1}(M)$ of $M$ and a sub-pseudomanifold $L$ of $\Pi^{1}(M)$ such that every point of $f(|\mathscr{B}(L)|)$ has a distance less than $\delta$ from $\bigcup_{j=1}^{p} \operatorname{Fr}\left(J_{j}\right)$ and

$$
\begin{aligned}
& \limsup _{r \rightarrow \infty} a\left(g^{(r)},|M| \sim|L|\right)<\varepsilon \cdot K^{-1}, \\
& \limsup _{r \rightarrow \infty} a\left(h^{(r)},|M| \sim|L|\right)<\varepsilon \cdot K^{-1} .
\end{aligned}
$$

By (2) one can choose a $\delta_{1}>0$ and a positive integer $r_{1}$ such that the sets

$$
U_{j}=\left\{x ; x \in R^{n} \text { and } d\left(x, F r\left(J_{j}\right)\right)<\delta_{1}\right\}
$$

are sufficiently small that

$$
a\left[g^{(r)}, f^{-1}\left(\bigcup_{j=1}^{p} U_{j}\right)\right]<\varepsilon \cdot K^{-1}
$$

and

$$
a\left[h^{(r)}, f^{-1}\left(\bigcup_{j=1}^{p} U_{j}\right)\right]<\varepsilon \cdot K^{-1}
$$

for all $r \geqq r_{1}$. Let $\Pi^{l}(M)$ be a subdivision of $M$ and $L$ a sub-pseudo-manifold of $\Pi^{l}(M)$ such that 
and

$$
|\mathscr{B}(L)| \subseteq f^{-1}\left(\bigcup_{j=1}^{p} U_{j}\right)
$$

$$
\begin{aligned}
& \underset{r \rightarrow \infty}{\limsup } a\left(g^{(r)},|M| \sim|L|\right)<\varepsilon \cdot K^{-1}, \\
& \limsup _{r \rightarrow \infty} a\left(h^{(r)},|M| \sim|L|\right)<\varepsilon \cdot K^{-1} .
\end{aligned}
$$

Let $\mathscr{M}_{1}$ be the middle space of the restriction of $f$ to $|L|, \eta$ be the inclusion mapping of $\mathscr{M}_{1}$ into $\mathscr{M}$ and $f=l_{1} \circ m_{1}$ be the monotone light factorization of $f$ with respect to $|L|$. Since

$$
\mathscr{E}_{1}=l_{1}^{-1}\left\{R^{n} \sim \bigcup_{j=1}^{p} U_{j}\right\}
$$

is a closed subset of $\mathscr{M}_{1}$ which does not intersect $m_{1}\{|\mathscr{B}(L)|\}$, there exists a continuous function $\chi_{1}$ on $\mathscr{M}_{1}$ such that

$$
\begin{aligned}
\chi_{1}(\zeta) & =\chi\{\eta(\zeta)\}, & & \zeta \in \mathscr{E}_{1} \\
& =0 & & \zeta \in m\{|\mathscr{B}(L)|\},
\end{aligned}
$$

and

$$
\left|\chi_{1}(\zeta)\right| \leqq K
$$

for all $\zeta \in \mathscr{M}_{1}$. By 4.2

$$
\lim _{r \rightarrow \infty}\left\{v\left(g^{(r)}\right)\right\}\left(\chi_{1} \circ m_{1}\right)=\lim _{r \rightarrow \infty}\left\{v\left(h^{(r)}\right)\right\}\left(\chi_{1} \circ m_{1}\right)
$$

But

$$
\left|\left\{v\left(g^{(r)}\right)\right\}\left(\chi \circ m-\chi_{1} \circ m_{1}\right)\right| \leqq 2 K a\left\{g^{(r)}, f^{-1}\left\{\bigcup_{j=1}^{p} U_{j}\right\} \cup(|M| \sim|L|)\right\},
$$

hence by (3) and (6),

$$
\underset{r \rightarrow \infty}{\limsup }\left|\left\{v\left(g^{(r)}\right)\right\}\left(\chi \circ m-\chi_{1} \circ m_{1}\right)\right|<2 \varepsilon
$$

and similarly

$$
\limsup _{r \rightarrow \infty}\left|\left\{v\left(h^{(r)}\right)\right\}\left(\chi \circ m-\chi_{1} \circ m\right)\right|<2 \varepsilon
$$

so that by (8)

$$
\mid \lim _{r \rightarrow \infty}\left\{v\left(g^{(r)}\right\}(\chi \circ m)-\lim _{r \rightarrow \infty}\left\{v\left(h^{(r)}\right)\right\}(\chi \circ m) \mid<4 \varepsilon\right.
$$

contradicting (1). 


\section{REFERENCES}

1. L. Cesari, Surface area, Annals of Mathematics Studies, No. 35, Princeton Univ. Press, Princeton, N. J., 1956.

2. H. Federer, Currents and area, Trans. Amer. Math. Soc. 98 (1961), 204-233.

3. H. Federer and W. H. Fleming, Normal and integral currents, Ann. of Math. (2) 72 (1960), 458-520.

4. W. Hurewicz and H. Wallman, Dimension theory, Princeton Univ. Press, Princeton, N. J., 1941.

5. S. Lefschetz, Algebraic topology, Amer. Math. Soc. Colloq. Publ., Vol. 27, Amer. Math. Soc., Providence, R. I., 1942.

6. J. H. Michael, Integration over parametric surfaces, Proc. London Math. Soc. (3) 7 (1957), 616-640.

7. - The convergence of measures on parametric surfaces, Abstract 576-84, Notices Amer. Math. Soc. 7 (1960), 920.

8. L. S. Pontrjagin, Foundations of combinatorial topology, Graylock Press, Rochester, N. Y., 1952.

9. H. Whitney, Geometric integration theory, Princeton Univ. Press, Princeton, N. J., 1957.

10. G. T. Whyburn, Analytic topology, Amer. Math. Soc. Colloq. Publ., Vol. 28, Amer. Math. Soc., Providence, R. I., 1942.

UNIVERSTTY OF ADELAIDE,

Adelaide, South Australia 